MATHEMATICS OF COMPUTATION

Volume 77, Number 261, January 2008, Pages 495-502

S 0025-5718(07)02002-9

Article electronically published on May 14, 2007

\title{
THE LUCAS-PRATT PRIMALITY TREE
}

\author{
JONATHAN BAYLESS
}

\begin{abstract}
In 1876, E. Lucas showed that a quick proof of primality for a prime $p$ could be attained through the prime factorization of $p-1$ and a primitive root for $p$. V. Pratt's proof that PRIMES is in NP, done via Lucas's theorem, showed that a certificate of primality for a prime $p$ could be obtained in $O\left(\log ^{2} p\right)$ modular multiplications with integers at most $p$. We show that for all constants $C \in \mathbb{R}$, the number of modular multiplications necessary to obtain this certificate is greater than $C \log p$ for a set of primes $p$ with relative asymptotic density 1 .
\end{abstract}

\section{INTRODUCTION}

Over a quarter of a century before Agrawal, Kayal, and Saxena showed PRIMES is in P in [1], V. Pratt showed in [11] that PRIMES is in NP by utilizing the following theorem of Lucas:

Theorem 1. Suppose $p>1$ is an odd integer and

$$
\left\{\begin{array}{l}
a^{\frac{p-1}{2}} \equiv-1 \bmod p \\
a^{\frac{p-1}{2 q}} \not \equiv-1 \bmod p \text { for every odd prime } q \mid p-1
\end{array}\right.
$$

Then $p$ is prime and $a$ is a primitive root of $p$. Conversely, if $p$ is an odd prime, then every primitive root a of $p$ satisfies conditions (1.1).

In 1877, Lucas [8] stated a result essentially equivalent to Theorem 1] it is based on his work from a year earlier. The actual statement presented here can be found in [3, Theorem 4.1.8].

To achieve this Lucas-Pratt certificate of primality, we would need to find a primitive root $a$ modulo $p$, and we would need to certify each of the primes $q$ appearing in the second condition. By charting this process, we find what is called a Lucas-Pratt tree. For example, to certify 9461 in this manner, we have the following tree:

Received by the editor June 26, 2006 and, in revised form, November 14, 2006.

2000 Mathematics Subject Classification. Primary 11Y16; Secondary 11 N37.

The author was supported by a Dartmouth Graduate Fellowship.

(C)2007 American Mathematical Society Reverts to public domain 28 years from publication 


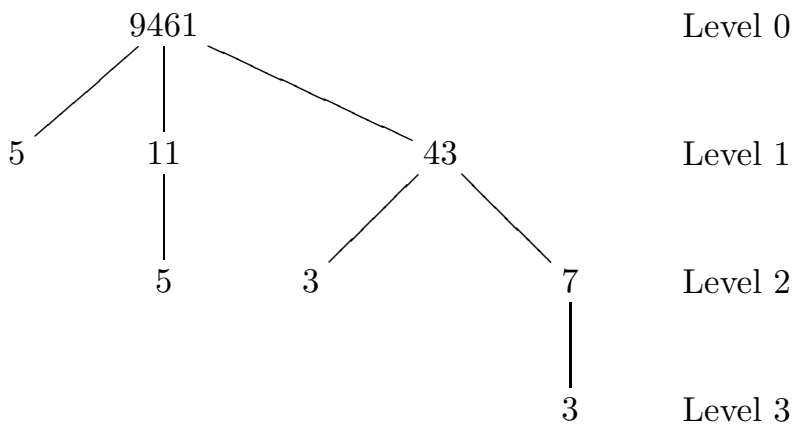

Notice that the branches beneath a prime $r$ correspond to the odd primes dividing $\varphi(r)=r-1$. Pratt showed that the number of primes appearing in the Lucas-Pratt tree for a prime $p$, denoted $N(p)$, is $O(\log p)$. In fact, he showed:

Theorem 2. $N(p)<\log _{2} p$.

Here $\log _{2} p$ denotes the base-2 logarithm of $p$.

Let $M(p)$ denote the number of modular multiplications of integers with size at most $p$ (using a standard modular exponentiation algorithm) needed to verify the primality of an integer $p$ by this method. As a corollary to the above theorem, Pratt showed:

Corollary 1. $M(p)=O\left(\log ^{2} p\right)$.

See [11] for details of either proof, or [3, Theorem 4.1.9] for a simple exposition of both results. It is clear that $M(p)$ is at least $\log _{2} p-2$, as this is a lower bound for the number of multiplications necessary to evaluate the first condition of Theorem 1

A relevant result of Pomerance is the following theorem (found in [10]):

Theorem 3. For every prime $p$ there is a proof that it is prime which requires for its verification $\left(\frac{5}{2}+o(1)\right) \log _{2} p$ multiplications modulo $p$.

Thus, in principle, for each prime $p$ there exists a primality proof of order $O(\log p)$, and so one might ask if Pratt's result can be improved to $M(p)=O(\log p)$.

For any positive integer $n$, let $\varphi_{k}(n)$ denote the $k$-th iterate of the Euler $\varphi$ function at $n$, and let $\varphi_{0}(n)=n$. Define $F(p)$ as the product of the distinct odd primes dividing $\prod_{k \geq 0} \varphi_{k}(p)$. An odd prime $q \neq p$ appears in level $k>1$ of the Lucas-Pratt tree for $p$ if it divides $\varphi(r)$ for some prime $r$ in level $k-1$ of the tree. Since this means that $q \mid \varphi_{k}(p)$, we have that $F(p)$ is equal to the squarefree product of the primes appearing in the Lucas-Pratt tree for $p$. As an example, $F(9461)=9461 \cdot 5 \cdot 11 \cdot 43 \cdot 3 \cdot 7=469880565$.

We first prove a theorem analogous to a result in [7] and suggested in the same paper. The proof of this theorem follows the same plan as in [7, but certain extra tools are brought into play.

Theorem 4. For each $\epsilon>0$, the set of primes $p$ for which

$$
\varphi(F(p))>p^{(1-\epsilon) \frac{\log \log p}{\log \log \log p}}
$$

has relative asymptotic density 1 within the set of all primes. 
We use this theorem to obtain the following result:

Theorem 5. Let $\epsilon>0$ be arbitrary and let $C(p)=(1-\epsilon) \log \log p / \log \log \log p$. Then $M(p)>C(p) \log _{2} p$ for a set of primes $p$ with relative asymptotic density 1 . In particular, for all $C \in \mathbb{R}, M(p)>C \log p$ for almost all primes $p$.

As noted in Pratt's paper, naive multiplication modulo $p$ has bit complexity $O\left(\log ^{2} p\right)$, and so, using naive multiplication, the bit complexity of the certificate of the prime $p$ is $O\left(\log ^{4} p\right)$. One might ask if the bit complexity can be bounded below in a manner analogous to what we do here with $M(p)$. Limited attempts to use the result of this paper to answer this question have met with little success, but it would be interesting to see if a similar lower bound on the bit complexity is possible.

In 2, the related question of the height of the Lucas-Pratt tree is considered by Banks and Shparlinski, who prove a lower bound on the height for almost all primes $p$.

Throughout, $p, q$, and $r$ will always be primes, and $n$ will always be a positive integer.

\section{TeChNicAl PREPARATion}

We now begin developing the tools we will use to prove Theorem 4

Lemma 1. For any value of $x \geq e^{e}$ and any prime $p<x$, the following holds:

$$
\sum_{\substack{r \leq \frac{x}{2} \\ r \equiv 1 \bmod p}} \frac{1}{r \log \frac{x}{r}} \leq \frac{30 \log \log x}{p \log \frac{x}{p}} .
$$

Proof. We use the Brun-Titchmarsh inequality of [9]; namely, for coprime integers $k$ and $\ell$, the number of primes $q \leq x$ with $q \equiv \ell \bmod k$, denoted $\pi(x ; k, \ell)$, satisfies

$$
\pi(x ; k, \ell) \leq \frac{2 x}{\varphi(k) \log \frac{x}{k}} \text { for } x>k .
$$

For prime $p \geq 2$ and $(\ell, p)=1$, we can use the fact that $\varphi(p)=p-1$ to see that

$$
\pi(x ; p, \ell) \leq \frac{2 x}{\varphi(p) \log \frac{x}{p}} \leq \frac{4 x}{p \log \frac{x}{p}} \text { for } x>p .
$$

Note that the first prime $r \equiv 1 \bmod p$ is at least $\frac{3 p}{2}$, so we need only consider $p \leq \frac{x}{3}$. We use partial summation to obtain

$$
\begin{aligned}
\sum_{\substack{r \leq \frac{x}{2} \\
r \equiv 1 \bmod p}} \frac{1}{r \log \frac{x}{r}} & =\frac{\pi\left(\frac{x}{2} ; p, 1\right)}{\frac{x}{2} \log 2}+\int_{\frac{3 p}{2}}^{\frac{x}{2}} \pi(t ; p, 1)\left(\frac{1}{t^{2} \log \frac{x}{t}}-\frac{1}{t^{2}\left(\log \frac{x}{t}\right)^{2}}\right) d t \\
& \leq \frac{4}{p \log \frac{x}{2 p} \log 2}+\int_{\frac{3 p}{2}}^{\frac{x}{2}} \frac{4}{p t \log \frac{t}{p} \log \frac{x}{t}} d t \\
& =\frac{4}{p \log \frac{x}{2 p} \log 2}+\frac{4}{p \log \frac{x}{p}} \int_{\frac{3 p}{2}}^{\frac{x}{2}}\left(\frac{1}{\log \frac{t}{p}}+\frac{1}{\log \frac{x}{t}}\right) \frac{d t}{t} \\
& =\frac{4}{p \log \frac{x}{2 p} \log 2}+\left.\frac{4}{p \log \frac{x}{p}} \cdot\left(\log \log \frac{t}{p}-\log \log \frac{x}{t}\right)\right|_{\frac{3 p}{2}} ^{\frac{x}{2}} .
\end{aligned}
$$


Since $\frac{4}{\log 2 \log \frac{x}{2 p}}<\frac{16}{\log \frac{x}{p}}$ and $16-4 \log \log 2-4 \log \log 3 / 2<22$, we see that

$$
\begin{aligned}
\sum_{\substack{r \leq \frac{x}{2} \\
r \equiv 1 \bmod p}} \frac{1}{r \log \frac{x}{r}} & \leq \frac{1}{p \log \frac{x}{p}}\left(22+8 \log \log \frac{2 x}{3 p}\right) \\
& \leq \frac{30}{p \log \frac{x}{p}} \log \log x,
\end{aligned}
$$

where for the last step we use $x \geq e^{e}$.

This allows us to prove the following proposition:

Proposition 1. For all $x \geq e^{e}$, each prime $p<x$, and all positive integers $k$, the number of primes $q \leq x$ with $p \mid \varphi_{k}(q)$ is bounded above by

$$
\frac{4 x}{p \log \frac{x}{p}}(31 \log \log x)^{k-1} .
$$

Proof. We prove the proposition by induction. For $k=1$, we have

$$
\sum_{\substack{q \leq x \\ p \mid \varphi(q)}} 1=\pi(x ; p, 1),
$$

and the result follows from (2.1).

For $k \geq 1$, assume the proposition holds up to $k$. Then if $p \mid \varphi_{k+1}(q)$, either $p^{2} \mid \varphi_{k}(q)$ or there exists a prime $r \equiv 1 \bmod p$ such that $r \mid \varphi_{k}(q)$. Thus,

$$
\sum_{\substack{q \leq x \\ p \mid \varphi_{k+1}(q)}} 1 \leq \sum_{\substack{q \leq x \\ p^{2} \mid \varphi_{k}(q)}} 1+\sum_{r \equiv 1 \bmod p} \sum_{\substack{q \leq x \\ r \mid \varphi_{k}(q)}} 1 .
$$

In the second term, $r$ is an odd prime and it divides the even number $\varphi_{k}(q)$, so $r \leq \frac{1}{2} \varphi_{k}(q) \leq \frac{1}{2} q \leq \frac{1}{2} x$. Hence, by the induction hypothesis and Lemma 1] we have

$$
\begin{aligned}
\sum_{\substack{q \leq x \\
p \mid \varphi_{k+1}(q)}} 1 & \leq \frac{4 x}{p \log \frac{x}{p}}(31 \log \log x)^{k-1}+\sum_{\substack{r \leq \frac{x}{2} \\
r \equiv 1 \bmod p}} \frac{4 x}{r \log \frac{x}{r}}(31 \log \log x)^{k-1} \\
& \leq \frac{4 x}{p \log \frac{x}{p}}(31 \log \log x)^{k-1}(1+30 \log \log x) \\
& \leq \frac{4 x}{p \log \frac{x}{p}}(31 \log \log x)^{k},
\end{aligned}
$$

where for the last inequality we use $x \geq e^{e}$. This proves the proposition.

We now state a lemma and a proposition from [7. Define $\Omega(n)$ to be the number of prime divisors of $n$, including multiplicity. We have the following lemma, the proof of which is suggested in Exercise 05 of [5]:

Lemma 2. There exists an absolute constant $C_{1}>0$ such that for each positive integer $k$,

$$
\sum_{\substack{n \leq x \\ \Omega(n) \geq k}} 1 \leq C_{1} \frac{k}{2^{k}} x \log x .
$$


Define

$$
F_{K}(n)=\prod_{0 \leq k \leq K} \varphi_{k}(n) .
$$

Proposition 2. There exists an absolute constant $C_{2}>0$ such that for all suffciently large numbers $x$, all numbers $y \geq 1$, and all integers $K \geq 1$, the number of integers $n \leq x$ with $p^{2} \mid F_{K}(n)$ for some prime $p>y$ is at most

$$
\frac{x}{y} K\left(C_{2} \log \log x\right)^{2 K}
$$

Clearly, the bound in the proposition still holds if we restrict the count to prime $n$. Using the arguments from [4] and [7] together with a short computation, we may take $C_{2}=27$.

We need one last proposition before moving on to the proof of the theorems.

Proposition 3. There exists an absolute constant $C_{3}>0$ such that for each $x \geq e^{e}$ and every positive integer $K$, the number of primes $p \leq x$ for which $\Omega\left(F_{K}(p)\right)>$ $2(6 \log \log x)^{K}$ is bounded above by

$$
C_{3} \frac{x}{\log ^{2} x}(31 \log \log x)^{K-1} .
$$

Proof. By Lemma 2 for any $t \in(1, x]$,

$$
\begin{aligned}
\sum_{\substack{q \leq t \\
1)>6 \log \log x}} 1 \leq \sum_{\substack{n \leq t-1 \\
\Omega(n)>6 \log \log x}} 1 \leq \frac{6 C_{1} \log \log x}{2^{6 \log \log x} t \log t} \\
\leq 6 C_{1} \frac{t \log \log x}{(\log x)^{6 \log 2-1}} \leq 6 C_{1} \frac{t \log \log x}{(\log x)^{3}} .
\end{aligned}
$$

Since

$$
6 C_{1} \frac{t \log \log x}{\log ^{3} x} \leq 6 C_{1} \frac{x}{\log ^{2} x},
$$

the proposition holds for $K=1$, provided $C_{3} \geq 6 C_{1}$; we now assume $K \geq 2$.

If $\Omega(q-1) \leq 6 \log \log x$ for each prime $q$ dividing $F_{K-1}(p)$, then for all integers $1 \leq k \leq K, \Omega\left(\varphi_{k}(p)\right) \leq(6 \log \log x)^{k}$ and $\Omega\left(F_{K}(p)\right) \leq 2(6 \log \log x)^{K}$. Therefore, if $\Omega\left(F_{K}(p)\right)>2(6 \log \log x)^{K}$, there exists a prime $q$ dividing $F_{K-1}(p)$ with $\Omega(q-1)>$ $6 \log \log x$. Clearly, such a $q$ must be odd, and since $F_{K-1}(p)=\prod_{k \leq K-1} \varphi_{k}(p)$, $q \mid \varphi_{k}(p)$ for some $k \leq K-1$. If this $k=0$, then $q=p$; if $k>0, \varphi_{k}(p)$ is even, so $q \leq \frac{x}{2}$. Hence, we need only consider either $q=p$ or $q \leq \frac{x}{2}$. Thus, by (2.2) and Proposition 1 we have that

$$
\begin{gathered}
\sum_{\substack{p \leq x\\
)>2(6 \log \log x)^{K}}} 1 \leq \sum_{\substack{q \leq x \\
\Omega(q-1)>6 \log \log x}} 1+\sum_{\substack{q \leq \frac{x}{2} \\
\Omega(q-1)>6 \log \log x}} \sum_{1 \leq k \leq K-1} \sum_{\substack{p \leq x \\
q \mid \varphi_{k}(p)}} 1 \\
\leq \frac{6 C_{1} x \log \log x}{\log ^{3} x}+\sum_{\substack{q \leq \frac{x}{2} \\
\Omega(q-1)>6 \log \log x}} \sum_{\substack{1 \leq k \leq K-1 \\
q}} \frac{4 x(31 \log \log x)^{k-1}}{q \log \frac{x}{q}} \\
\leq \frac{6 C_{1} x \log \log x}{\log ^{3} x}+\sum_{\substack{q \leq \frac{x}{2} \\
\Omega(q-1)>6 \log \log x}} \frac{5 x(31 \log \log x)^{K-2}}{q \log \frac{x}{q}} .
\end{gathered}
$$


Approaching this last sum with partial summation, increasing the range of the sum to $q \leq x$, and using the bound in (2.2), we have

$$
\begin{aligned}
\sum_{\substack{q \leq \frac{x}{2} \\
\Omega(q-1)>6 \log \log x}} \frac{1}{q \log \frac{x}{q}} & <\sum_{\substack{q \leq x \\
\Omega(q-1)>6 \log \log x}} \frac{2}{q} \\
& \leq \frac{6 C_{1} x \log \log x}{\log ^{3} x} \cdot \frac{2}{x}+\int_{3}^{x} \frac{6 C_{1} t \log \log x}{\log ^{3} x} \cdot \frac{2}{t^{2}} d t \\
& \leq \frac{12 C_{1} \log \log x}{\log ^{2} x} .
\end{aligned}
$$

Putting this together, we have that

$$
\begin{aligned}
\sum_{\substack{p \leq x \\
>2(6 \log \log x)^{K}}} 1 & \leq \frac{6 C_{1} x \log \log x}{\log ^{3} x}+\frac{2 C_{1} x(31 \log \log x)^{K-1}}{\log ^{2} x} \\
& \leq \frac{6 C_{1} x(31 \log \log x)^{K-1}}{\log ^{2} x},
\end{aligned}
$$

so we may take $C_{3}=6 C_{1}$, which proves the proposition.

\section{The proof of Theorem 4}

We are now ready to prove that $\varphi(F(p))$ grows faster than any fixed power of $p$ for almost all primes $p$.

Proof. We follow the plan of Theorem 3 in [7. Let $x>e^{e}$ and fix $0<\epsilon<1$ arbitrarily small. Set

$$
K=\lceil(1-\epsilon) \log \log x / \log \log \log x\rceil .
$$

For $p \leq x$ prime, factor $F_{K}(p)=A \cdot B$, where $A$ is the product of the primes dividing $F_{K}(p)$ which are less than or equal to $\log ^{3} x$, and $B$ is the product of the primes larger than $\log ^{3} x$ (including multiplicity in both $A$ and $B$ ). Proposition 3 gives that with at most $O\left(\frac{x}{\log ^{2} x}(31 \log \log x)^{K-1}\right)=o(\pi(x))$ exceptions, all choices of $p$ have

$$
A \leq\left(\log ^{3} x\right)^{2(6 \log \log x)^{K}}=\exp \left((6 \log \log x)^{K+1}\right)=x^{o(1)} .
$$

For $x$ sufficiently large, the minimal order of $\varphi(m) / m$ for $m \leq x$ (see [6. Theorem $328])$ gives that $\varphi_{j+1}(p) / \varphi_{j}(p)>(2 \log \log x)^{-1}$ holds for each $j \in[0, K-1]$. Since $\pi\left(\frac{x}{2 \log \log x}\right)=o(\pi(x))$, we may assume $p>x /(2 \log \log x)$. Then,

$$
\begin{aligned}
F_{K}(p) & =p^{K+1} \prod_{i=0}^{K} \frac{\varphi_{i}(p)}{p}=p^{K+1} \prod_{i=1}^{K} \prod_{j=0}^{i-1} \frac{\varphi_{j+1}(p)}{\varphi_{j}(p)} \\
& >p^{K+1} /(2 \log \log x)^{1+2+\cdots+K}=p^{K+1} /(2 \log \log x)^{K(K+1) / 2} \\
& >x^{K+1} /(2 \log \log x)^{(K+1)(K+2) / 2}>x^{K+1 / 2} .
\end{aligned}
$$

So with $o(\pi(x))$ exceptions, all choices of $p \leq x$ have

$$
B=\frac{F_{K}(p)}{A}>x^{K+1 / 4} \text {. }
$$


By Proposition 2. for sufficiently large $x$, the number of primes $p \leq x$ such that $q^{2} \mid F_{K}(p)$ for some $q \mid B$ is bounded above by

$$
\begin{aligned}
\frac{x}{\log ^{3} x} \frac{(\log \log x)(\log \log x)^{2 K}}{\log \log \log x}\left(C_{2}\right)^{2 K} & \leq \frac{x}{\log ^{3} x}(\log \log x)^{2 K+1}\left(C_{2}\right)^{2 K} \\
& \leq \frac{x}{\log ^{3} x}(\log x)^{2-2 \epsilon}(\log \log x)^{3}\left(C_{2}\right)^{2 K} \\
& =\frac{x}{(\log x)^{1+2 \epsilon+o(1)}} \\
& =o(\pi(x)) .
\end{aligned}
$$

So, but for $o(\pi(x))$ choices of the prime $p \leq x, B$ is squarefree. Since $B \mid F(p)$, we have that $\varphi(B) \mid \varphi(F(p))$. Again applying the minimal order of the Euler $\varphi$-function, we have

$$
\varphi(B)>\frac{B}{2 \log \log B}>\frac{x^{K+1 / 4}}{2(\log (K+1 / 4)+\log \log x)}>\frac{x^{K+1 / 4}}{3 \log \log x}>x^{K}
$$

for sufficiently large $x$. Thus, with at most $o(\pi(x))$ exceptions, $\varphi(F(p))>x^{K} \geq p^{K}$ holds for primes $p \leq x$. This completes the proof of Theorem 4 .

Corollary 2. For almost all primes $p, F(p)$ grows faster than any fixed power of $p$.

\section{LuCAS-Pratt trees}

Now to attain our primary result, we will use Pratt's Theorem on $N(p)$, the number of primes appearing in the Lucas-Pratt tree for a prime $p$.

Proof. For each prime $q$ appearing in the Lucas-Pratt tree for $p$, we have to at least show that $a^{\frac{q-1}{2}} \equiv-1 \bmod q$ for some primitive root $a$. This takes at least $\log _{2}\left(\frac{q-1}{2}\right)$ modular multiplications $\bmod q$. Thus,

$$
M(p) \geq \sum_{q \mid F(p)} \log _{2}\left(\frac{q-1}{2}\right)=\sum_{q \mid F(p)}\left(\log _{2}(q-1)-1\right) \geq \log _{2} \varphi(F(p))-N(p) .
$$

Thus by Theorems 2 and 4 above, for each fixed $\epsilon>0$, but for $o(\pi(x))$ primes $p \leq x$

$$
\begin{aligned}
M(p) & >\frac{1-\epsilon}{\log 2} \frac{\log p \log \log p}{\log \log \log p}-\frac{1}{\log 2} \log p \\
& =\frac{1}{\log 2}\left((1-\epsilon) \frac{\log \log p}{\log \log \log p}-1\right) \log p .
\end{aligned}
$$

Since $\epsilon>0$ is arbitrary, this completes the proof of Theorem 5

\section{ACKNOWLEDGMENTS}

I would like to thank my advisor, Carl Pomerance, for his guidance on this work and extensive help in preparing this manuscript. I would also like to thank Hugh Williams for his helpful communication regarding Lucas's Theorem and an anonymous referee for some helpful comments. 


\section{REFERENCES}

1. M. Agrawal, N. Kayal, and N. Saxena, PRIMES is in P, Annals of Mathematics, 160 (2004), 781-793. MR2123939 (2006a:11170)

2. W. Banks and I. Shparlinski, On values taken by the largest prime factor of shifted primes, Journal of the Australian Mathematical Society, 82 (2007), 133-147.

3. R. Crandall and C. Pomerance, Prime Numbers: A Computational Perspective, SpringerVerlag, New York, 2001. MR.1821158(2002a:11007)

4. P. Erdős, A. Granville, C. Pomerance, and C. Spiro, On the normal behavior of the iterates of some arithmetic functions, in: Analytic Number Theory, Proceedings of a Conference in Honor of P. T. Bateman, Birkhäuser; Boston, Inc. 1990, 165-204. MR.1084181 (92a:11113)

5. R. Hall and G. Tenenbaum, Divisors, Cambridge University Press, Cambridge, 1988. MR 964687 (90a:11107)

6. G. Hardy and E. Wright, An Introduction to the Theory of Numbers, 4th ed., Clarendon Press, Oxford, UK, 1971. MR568909 (81i:10002)

7. F. Luca and C. Pomerance, Irreducible radical extensions and Euler-function chains, Integers, to appear, (Available at http://math.dartmouth.edu/ carlp/PDF/radicalpaper5.pdf).

8. E. Lucas, Considérations nouvelles sur la théorie des nombres premiers et sur la division géométrique de la circonférence en parties égales, Association Française pour l'Avancement des Sciences, Comptes Rendus, 6 (1877), 159-167.

9. H. Montgomery and R. Vaughan, The large sieve, Mathematika (20) 40 (1973), 119-134. MR0374060 (51:10260)

10. C. Pomerance, Very short primality proofs, Mathematics of Computation (48) $17 \mathbf{7}$ (1987), 315-322. MR 866117 (88b:11088)

11. V. Pratt, Every prime has a succinct certificate, SIAM Journal on Computing, 4 (1975), 214-220. MR0391574(52:12395)

Department of Mathematics, Dartmouth College, Hanover, New Hamphshire 037553551

E-mail address: jonathan.bayless@dartmouth.edu 